ISSN 0103-5150

Fisioter. Mov., Curitiba, v. 26, n. 1, p. 159-166, jan./mar. 2013

Licenciado sob uma Licença Creative Commons

\title{
Prevalência de bruxismo e distúrbio do sono em deficientes visuais
}

\author{
Prevalence of bruxism and sleep disorders in \\ the visually impaired individuals
}

\section{Bárbara Bernardo Rinaldo da Silva ${ }^{[a]}$, Kátia Roberta Rapôso Sales Lacerda ${ }^{[b]}$, Ana Paula de Lima Ferreira ${ }^{[\mathrm{c}]}$, Marcelo de Souza Figueiroa ${ }^{[\mathrm{d}]}$}

[a] Fisioterapeuta graduada pela Faculdade Integrada do Recife (FIR), pós-graduanda em Fisioterapia Pediátrica pelo Instituto Materno Infantil de Pernambuco (IMIP), Recife, PE - Brasil, e-mail: barbara_bernardo@hotmail.com

[b] Fisioterapeuta graduada pela Faculdade Integrada do Recife (FIR), Recife, PE - Brasil, e-mail: katynha87@gmail.com

[c] Fisioterapeuta, mestre em Nutrição, docente do curso de Fisioterapia da Universidade Federal de Sergipe, São Cristóvão, SE - Brasil, e-mail: apllima@yahoo.com.br

[d] Fisioterapeuta, especialista em Morfologia e docente do Curso de Fisioterapia da Faculdade Estácio (FIR), Recife, PE Brasil, e-mail: marcelobaikal@hotmail.com

\section{Resumo}

Introdução: 0 termo "bruxismo" significa ranger ou apertar os dentes sem objetivos funcionais aparentes; é um distúrbio que ocorre usualmente durante o sono e geralmente está associado a microdespertares. Os deficientes visuais estão mais predispostos a apresentarem distúrbio do sono em razão de uma possível disfunção da glândula pineal, alterando a produção de melatonina, e, consequentemente, por apresentarem sintomas de bruxismo relacionado à ausência da percepção de luz. Objetivo: Verificar a ocorrência de bruxismo e distúrbio do sono em deficientes visuais. Materiais e métodos: Amostra de conveniência com casuística de 63 voluntários com acuidade visual inferior a 0,05 (20/400) até a ausência da percepção luminosa. Foram submetidos à aplicação do Índice de Qualidade do Sono de Pittsburgh (PSQI) e do Inquérito para Diagnóstico de Bruxismo. Resultados: A prevalência de bruxismo e má qualidade do sono foram, respectivamente, de $80,95 \%$ e de $85,71 \%$. O bruxismo foi mais frequente entre aqueles com história de deficiência visual congênita $(65,08 \%)$ do que entre os portadores de deficiência visual adquirida $(34,92 \%)$, não sendo encontrada diferença significante quando comparados homens e mulheres. Já a ocorrência de má qualidade do sono foi similar entre portadores de deficiência visual congênita e adquirida. 
Foi verificada a associação positiva entre bruxismo e má qualidade do sono. Conclusão: Pôde-se verificar uma alta ocorrência de bruxismo e distúrbio do sono na população de deficientes visuais pesquisada. Este estudo tem importante contribuição epidemiológica e os seus resultados poderão ser utilizados para delinear futuras intervenções terapêuticas.

Palavras-chave: Bruxismo. Distúrbio do sono. Deficiência visual.

\section{Abstract}

Introduction: The word "bruxism" means grinding or clenching your teeth without apparent functional objectives that usually occurs during sleep, and this generally associated with arousals. Visually impaired people are more likely to submit sleep disorder due to a possible pineal gland dysfunction by altering melatonin production and therefore the present bruxism symptoms related to the absence of perception of light. Objective: To verify the bruxism occurrence and sleep disturbance within visually impaired individuals. Materials and methods: Convenience sampling case-by-case of 63 volunteers with visual acuity under 0.05 (20/400) down to absence of visual perception. Submitted to Pittsburgh Sleep Quality Index (PSQI) test and Bruxism Diagnostic Enquiry. Results: The prevalence of bruxism and low quality sleep were, respectively, of $80.95 \%$ and $85.71 \%$. Bruxism was more prevalent in those with congenital visual impairment (65.08\%) than those with acquired visual impairment (34.92\%), with no significant statistical difference between men and women. However, the occurrence of low quality sleep was similar between those with congenital and acquired visual impairment. It was verified the positive relation with bruxism and low quality sleep. Conclusion: It was possible to verify a high occurrence of bruxism and sleep disturbance in the surveyed population. This study has major epidemiological contribution and its results might be used to guide further therapeutic interventions.

Keywords: Bruxism. Sleep disturbance. Visually impaired.

\section{Introdução}

O termo "bruxismo" deriva da palavra grega brychein, que significa "ranger ou apertar os dentes" (1). 0 bruxismo é um dos distúrbios que mais contribuem para o desgaste dos dentes, doença periodontal e distúrbios temporomandibulares (DTM) (2), que pode ocorrer tanto em vigília (briquismo) como durante o sono (bruxismo propriamente dito) (3).

0 bruxismo pode ser definido também como hábito inconsciente, e às vezes consciente, de ranger, apertar e deslizar os dentes sem objetivos funcionais aparentes que ocorre usualmente durante o sono, geralmente associado a microdespertares; relaciona-se também a movimentos, parafunção orofacial, tique ou automatismo $(4,5,6)$.

Entre $85 \%$ e $90 \%$ das pessoas relatam episódios de ranger de dentes ao longo dos anos de suas vidas, mas a prevalência desse fenômeno na população geral é imprecisa e subestimada porque a maioria dos estudos epidemiológicos de bruxismo é baseada em populações e metodologias diferentes, a partir de relatos subjetivos; além disso, utilizam diferentes definições clínicas e sintomatológicas. Um dos problemas para o seu diagnóstico é o fato de que apenas $5 \%$ a $20 \%$ dos pacientes com bruxismo do sono (BS) tomam consciência dos episódios de ranger de dentes $(7,8,9)$.

Aproximadamente $80 \%$ das pessoas diagnosticadas com BS apresentam sintomas de sono fragmentado, sonolência excessiva diurna de moderada a intensa, sono não restaurador, despertares noturnos, sudorese ou pesadelos $(7,8)$. 0 ranger de dentes durante o sono, portanto, poderia ser considerada uma atividade oromotora excessiva, secundária ao microdespertar.

São escassos os estudos sobre a prevalência de bruxismo e distúrbio do sono (DS) em deficientes visuais. Dentre os poucos trabalhos publicados, destaca-se o estudo de Tabandeh et al. (10), que observou DS em população específica de deficientes visuais (quase $50 \%$ dos indivíduos cegos) e atribuiu a gravidade do DS à ausência da percepção de luz. Ele também observou que os principais problemas relacionados ao sono entre os indivíduos cegos foram: sono interrompido, seguido por aumento da latência do sono, curta duração do sono, 
e cochilos diurnos. A deficiência visual tem sido abordada de várias formas, no entanto, segundo a Organização Mundial de Saúde (OMS), a cegueira para fins epidemiológicos é definida como a acuidade visual inferior a 0,05 (20/400) até a ausência da percepção luminosa (11).

A relação entre deficiência visual e distúrbio do sono foi explorada neste estudo por conveniência, baseada no fato de que os deficientes visuais estão mais predispostos a apresentarem distúrbio do sono causado por possível disfunção da glândula pineal, alterando a produção de melatonina. A melatonina exerce um efeito de sincronização no marcador circadiano, sendo fortemente suprimida na presença de luz, aumentando até um determinado platô durante o sono e diminuindo novamente com o despertar (12, 13, 14). 0 ciclo claro-escuro é considerado um dos mais importantes fatores ambientais sincronizadores dos ritmos biológicos, já que a luz muda a fase do relógio circadiano por uma cascata de eventos (15).

Assim, o objetivo desta pesquisa foi verificar a relação entre Bruxismo e distúrbio do sono em deficientes visuais de uma instituição do Recife.

\section{Materiais e métodos}

Local de estudo e seleção da amostra

O estudo foi do tipo descritivo, transversal, realizado na Associação Beneficente dos Cegos do Recife (ASSOBECER), localizada na Estrada dos Remédios, Recife (PE).

Após aprovação pelo Comitê de Ética em Pesquisa da FIR (n. 21), a pesquisa foi realizada com uma amostra de 63 voluntários. Os questionários foram aplicados individualmente por uma das pesquisadoras em uma sala da própria instituição, na presença de uma funcionária para dar mais segurança aos deficientes visuais. Os participantes da pesquisa assinaram o Termo de Consentimento Livre e Esclarecido através da impressão digital ou de forma convencional (por extenso), com o uso do assinador.

Foram classificados indivíduos com acuidade visual inferior a 0,05 (20/400) até a ausência da percepção luminosa, de ambos os sexos, na faixa etária de 18 a 60 anos (indivíduos que já atingiram a maioridade e que ainda não fazem parte da população idosa) e cadastrados na Associação Beneficente dos Cegos do Recife (ASSOBECER).
Foram excluídos indivíduos com alterações neurológicas, auditivas e com história de depressão, ou que não aceitem fazer parte da pesquisa.

\section{Cálculo Amostral e Procedimentos}

Para o cálculo da amostra, utilizou-se o software EPI-INFO versão 6.0, considerando-se uma prevalência baseada na literatura de $27 \%$, erro máximo de $5 \%$ e perda de $25 \%$. 0 estudo foi realizado mediante a aplicação do Inquérito para Diagnóstico de Bruxismo (16) e do Índice de Qualidade do Sono de Pittsburgh (17) nos indivíduos cadastrados na ASSOBECER que atenderam aos critérios de inclusão da pesquisa.

As avaliações foram feitas coletando-se dados pessoais, tais como: nome, idade, ocupação, endereço e telefone para contato. 0 diagnóstico de Bruxismo foi baseado nos critérios epidemiológicos de Molina et al. (16). A população foi classificada como bruxômanos ou não bruxômanos a partir da interpretação de seis perguntas simples e objetivas; a pontuação zero significava ausência de bruxismo, 1 a 2 pontos bruxismo leve, 3 a 4 pontos bruxismo moderado e 5 a 6 pontos bruxismo severo.

A presença de DS foi investigada com o uso do Índice de Qualidade do Sono de Pittsburgh, constituído de 18 itens de autoavaliação da qualidade geral de sono, provendo informações sobre o número de horas efetivas de sono, número de perturbações de sono, latência do sono, eficiência do sono e uso de medicamento para dormir. A população estudada foi classificada de acordo com a pontuação que lhe foi dada (17). A soma da pontuação máxima desse instrumento é de 21 pontos, sendo os escores superiores a cinco pontos indicativos de qualidade ruim no padrão de sono (18).

\section{Análises estatísticas}

Foi utilizada estatística descritiva com valores relativos e absolutos para verificar a prevalência de bruxômanos, bem como para avaliar a qualidade do sono. Para comparar a média do índice de qualidade do sono entre bruxômanos e não bruxômanos, realizou-se o teste $t$ de Student para amostras independentes. Para avaliar se existe uma associação entre bruxismo e distúrbio do sono, bem como a relação entre bruxismo e idade, bruxismo e gênero, realizou-se o teste de 
associação de exato de Fisher ou qui-quadrado. Todos os testes foram realizados com nível de significância estabelecido em 5\%. 0 software utilizado para as análises foi o GraphPad Prism versão 3.0.

\section{Resultados}

Foram entrevistados 63 indivíduos, sendo 37 $(58,74 \%)$ do gênero masculino. A faixa etária da população foi de 19-60 anos (43,11 $\pm 11,94$ anos).

Dos pacientes com bruxismo, o grau leve foi o mais encontrado com 35 casos $(68,62 \%)$, seguida por moderado com 15 casos $(29,41 \%)$ e 1 caso severo $(1,96 \%)$. A qualidade do sono foi avaliada como ótima em 9 indivíduos (14,28\%), bordeliner em $10(15,88 \%)$ e pobre em 44 indivíduos (69,84\%) entrevistados. As frequências absolutas e relativas das variáveis em estudo estão apresentadas no Quadro 1.

Quadro 1 - Frequências absolutas e relativas das variáveis apresentadas pela amostra em estudo

(Continua)

\begin{tabular}{lcc}
\hline Variáveis & $\mathbf{n}$ & $\%$ \\
\hline Gênero & & 58,74 \\
\hline Masculino & 37 & 41,26 \\
Feminino & 26 & \\
\hline Idade (anos) & & \\
\hline $19-30$ & 12 & 19,04 \\
31-40 & 11 & 17,46 \\
41-50 & 16 & 25,40 \\
51-60 & 24 & 38,10 \\
\hline Bruxismo & & \\
\hline Bruxismo Leve & 35 & 68,62 \\
Bruxismo Moderado & 15 & 29,41 \\
Bruxismo Severo & 1 & 1,96 \\
Sem Bruxismo & 12 & 19,05 \\
\hline Qualidade do Sono & & \\
\hline Ótimo & 9 & 14,28 \\
Borderline & 10 & 15,88 \\
Pobre & 44 & 69,84 \\
\hline Perda visual & & \\
\hline Congênita & 41 & 65,08 \\
Adquirida & 22 & 34,92 \\
\hline & & \\
\hline
\end{tabular}

Analisando-se o tipo de deficiência visual, foi possível verificar que a ocorrência de bruxismo foi mais frequente entre aqueles com história de deficiência visual congênita $(65,08 \%)$ do que entre os portadores de deficiência visual adquirida $(34,92 \%)$. A ocorrência de má qualidade do sono foi similar entre portadores de deficiência visual congênita e adquirida.

De acordo com a classificação da perda visual, relatada de forma subjetiva pelos voluntários, verificou-se que $28(44,44 \%)$ tinham a percepção luminosa.

A prevalência de bruxismo, a má qualidade do sono e o percentual da associação positiva entre os distúrbios foram, respectivamente, de 80,95\%, 85,71\% e 87,50\% (Gráfico 1).

Embora tenha ocorrido uma maior frequência de bruxismo entre os indivíduos do gênero feminino, não foi encontrada diferença significante quando comparados homens e mulheres (Quadro 2). Por outro lado, foi verificada a associação positiva entre bruxismo e má qualidade do sono (Quadro 3).

Quadro 1 - Frequências absolutas e relativas das variáveis apresentadas pela amostra em estudo

\begin{tabular}{lcc} 
& & (Conclusão) \\
\hline Variáveis & $\mathbf{n}$ & \% \\
\hline Percepção luminosa & & \\
\hline Ausente & 35 & 55,56 \\
Presente & 28 & 44,44 \\
\hline
\end{tabular}

Fonte: Dados da pesquisa.

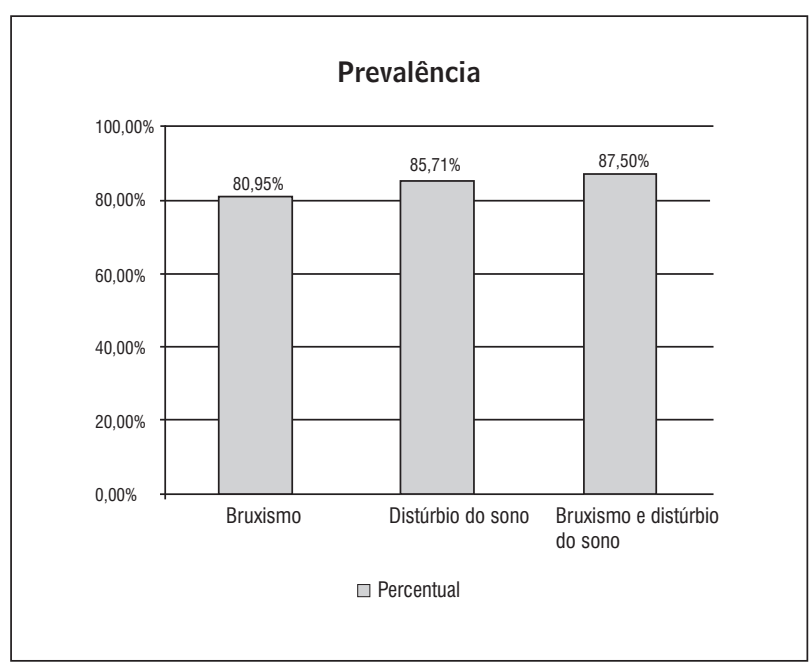

Gráfico 1 - Percentuais das prevalências de cada distúrbio e a associação entre eles

Fonte: Dados da pesquisa. 
Quadro 2 - Distribuição do gênero entre os sujeitos com e sem Bruxismo

\begin{tabular}{lccc}
\hline Variáveis & \multicolumn{2}{c}{ Bruxismo } & \\
\cline { 2 - 4 } & $\begin{array}{c}\text { Presente } \\
\mathbf{n}(\%)\end{array}$ & $\begin{array}{c}\text { Ausente } \\
\mathbf{n}(\%)\end{array}$ & p-valor \\
\hline Gênero & $28(75,68 \%)$ & $9(24,32 \%)$ & Teste Qui-Quadrado \\
Masculino & $23(88,46 \%)$ & $3(11,54 \%)$ & $0,970)$ \\
Feminino & 23 & \\
\hline
\end{tabular}

Fonte: Dados da pesquisa.

Quadro 3 - Distribuição da qualidade do sono entre os sujeitos com e sem Bruxismo

\begin{tabular}{lccc}
\hline & \multicolumn{2}{c}{ Bruxismo } & \\
\cline { 2 - 4 } Variáveis & $\begin{array}{c}\text { Presente } \\
\mathbf{n}(\%)\end{array}$ & $\begin{array}{c}\text { Ausente } \\
\mathbf{n}(\%)\end{array}$ & p-valor \\
\hline Qualidade do sono & & $5(55,56 \%)$ & \\
Ótimo & $4(44,44 \%)$ & $7(12,5 \%)$ & $\begin{array}{c}\text { Teste Qui-Quadrado } \\
(\mathbf{p}=0,0002)\end{array}$ \\
Borderline/Pobre & $49(87,5 \%)$ & & \\
\hline
\end{tabular}

Fonte: Dados da pesquisa.

\section{Discussão}

A escassez de estudos sobre prevalência de bruxismo e distúrbio do sono em deficientes visuais implicou na dificuldade em confrontar os dados deste trabalho com a literatura.

A maior prevalência de bruxismo encontrada na população em geral foi referida nos estudos de Branco et al. (19), que mostraram 55,5\% de ocorrência. Nesse estudo, a prevalência de bruxismo em deficientes visuais esteve bem acima desse percentual, sendo de $80,95 \%$. A maior prevalência de bruxismo em deficientes visuais pode estar relacionada ao fato de que esses indivíduos apresentam maiores níveis de ansiedade $(20,21)$, relacionados à necessidade de estarem continuamente alerta, a fim de evitar acidentes e incidentes.

Em relação à prevalência de distúrbio do sono, Verri et al. (22), utilizando o mesmo inquérito de Pittisburg, encontraram uma prevalência de 50\% de distúrbio de sono na população geral. Estudo preliminar usando o PSQI relatou uma prevalência estimada de distúrbio do sono em 58\% da população cega (10).
A prevalência de distúrbio do sono, neste estudo, foi de $85,71 \%$. Essa prevalência aumentada poderia ser explicada pela alteração do ritmo circadiano, que diminui a produção da melatonina.

Segundo Tabandeh et al. (10), a perda visual realmente pode exercer influência sobre os distúrbios do ritmo circadiano, mas os atributos qualitativos da perda visual não se correlacionam com a gravidade da perturbação do sono.

Distúrbios do sono são de origem multifatorial, sendo influenciados pelo emocional e por fatores psicológicos, rotina diária e atividade física e social. No entanto, é provável que um número relevante de indivíduos que sofram de problemas de sono tenha uma ampla alteração do ritmo circadiano. A análise mais detalhada dos padrões do sono pode permitir a melhor categorização de suas características em relação à dessincronização circadiana (10).

Analisando a presença ou ausência da percepção luminosa, foi verificado que, dos 63 deficientes visuais entrevistados, 28 (44,44\%) apresentaram presença de percepção luminosa. No entanto, não houve diferença estatística entre os indivíduos que não possuíam resquício visual e os que 
possuíam. A relação do ritmo circadiano e a percepção luminosa não foi objeto de estudo do presente trabalho. Contudo, Lockley et al. (23) estudaram 49 pacientes cegos e mostraram que o tipo de ritmo circadiano está diretamente relacionado à quantidade de percepção luminosa.

O distúrbio do sono presente em indivíduos com deficiência visual foi justificado por Leger et al. (24) como decorrente de estresse diário, que contribui para o aumento da latência do sono e despertar de manhã cedo, fato que é comum em deficientes visuais.

No presente estudo, o bruxismo foi mais frequente entre aqueles com história de deficiência visual congênita $(65,08 \%)$ do que entre aqueles com deficiência visual adquirida (34,92\%), não sendo encontrada diferença estatisticamente significante quando comparados homens e mulheres. Já a ocorrência de má qualidade do sono foi similar entre deficientes visuais congênitos e adquiridos.

Sabe-se que o distúrbio do sono é um fator de risco associado ao bruxismo $(3,6,25,26)$. Os resultados encontrados revelaram que dos $51(80,95 \%)$ indivíduos cegos que apresentavam bruxismo, 49 (87,5\%) tinham também má qualidade do sono. Portanto, foi verificada a associação positiva entre bruxismo e má qualidade do sono.

Vários estudos com indivíduos não cegos mostraram uma maior prevalência de bruxismo na população feminina $(4,27,28,29)$. Entretanto, um estudo de três semanas conduzido por Watanabe et al. (30) encontrou escores médios de bruxismo significativamente maiores em homens do que em mulheres. Diferentemente dessas pesquisas, não foram verificadas diferenças significativas em relação ao gênero no presente estudo.

Em relação às queixas encontradas, este estudo corrobora com Waller et al. (31), que descreveu a presença de insônia periódica, atraso no início do sono, curta duração do sono e o aumento de despertares espontâneos como queixas principais de indivíduos com má qualidade de sono.

\section{Conclusão}

Pôde-se verificar uma alta ocorrência de bruxismo e distúrbio do sono na população pesquisada. Este estudo tem importante contribuição epidemiológica visto que o tema não está amplamente explorado na literatura e os seus resultados poderão ser utilizados para delinear futuras intervenções terapêuticas.

\section{Referências}

1. Faot F, Custódio LG, Melo ACM, Hermann C. Bruxismo: aspectos históricos, conceitos, epidemiologia e fatores de risco. [dissertação]. Curitiba: Instituto Latino-Americano de Pesquisa e Ensino Odontológico; 2007.

2. Molina OF, Gaio DC, Cury MDN, Cury SE, Gimenez SEM, Salomão EC, et al. Uma análise crítica dos sistemas de classificação sobre o bruxismo: implicações com o diagnóstico, severidade e tratamento dos sinais e sintomas de DTM associados com o hábito. JBA. 2002;2 (5):61-9.

3. Coelho JPS, Lucena SC, Carvalho ALA, Lopes FF, Oliveira AEF. Bruxismo do sono e sua associação com distúrbios do sono em policiais. Ciênc Odontol Bras. 2009;12(1):31-6.

4. Gonçalves LPV, Toledo OA, Otero SAM. Relação entre bruxismo, fatores oclusais e hábitos bucais. Dental Press J Orthod. 2010;15(2):97-104. doi:10.1590/ S2176-94512010000200013.

5. Molina OF, Peixoto MGS, Santos ZC, Penoni JS, Aquilino RN, Peixoto MAS. Bruxism as mechanism subserving hysteria: a new theory. Rev Neurocienc. 2008; 16(4):262-8.

6. Ohayon MM, Li KK, Guilleminault MD. Risk factors for sleep bruxism in the general population. Chest J. 2001;119(1):53-61. doi:10.1378/chest.119.1.53.

7. Bader G, Lavigne G. Sleep bruxism: an overview of an oromandibular sleep movement disorder. Sleep Medicine Reviews. 2000; 4:27-43. doi:10.1053/ smrv.1999.0070.

8. Lavigne GJ, Manzini C. Bruxism. In: Kryger MH, Roth T, Dement WC. Principles and practice of sleep medicine. 3rd ed. Philadelphia: WB Saunders; 2000. PMCid:2144542.

9. Hublin C, Kaprio J, Partinen M, Koskenvou M. Sleep bruxism based on a self-report in a nationwide twin cohort. J Sleep Res. 1998;7:61-7. doi:10.1046/j.1365-2869.1998.00091.x. 
10. Tabandeh H, Lockley SW, Buttery R, Skene DJ, Defrance R, Arendt J, et al. Disturbance of sleep in blindness. Am J Ophthalmol. 1998;126(5):707-12. doi:10.1016/ S0002-9394(98)00133-0.

11. Reis PAC, Campos CMC, Fernandes LC. Características da população portadora de visão subnormal do Hospital São Geraldo: um estudo retrospectivo de 435 casos. Rev Bras Oftalmol. 1998;57(4):287-94.

12. Weinert D. Age-dependent changes of the circadian system. Chronobiology International 2000;17(3):261283. doi:10.1081/CBI-100101048.

13. Koller DE, Turek FW. Circadian rhythms and sleep in aging rodends. In: Hof PR, Mobbs CV. Functional neurobiology of aging. San Diego: Academic Press; 2001. p. 855-868.

14. Bianchin MM, Walz R, Spanis CW. Estudo do sono e de seus distúrbios. In: Kapczinsk F, Quevedo J, Izquierdo I. Bases biológicas dos transtornos psiquiátricos. Porto Alegre: Artmed; 2000.

15. Geib LTC, Cataldo A Neto, Wainberg RN, Nunes ML. Sono e envelhecimento. Rev Psiquiatr. 2003;25(3):453-65.

16. Molina OF, dos Santos Junior, Nelson SJ, Nowlin T. A clinical study of specific signs and symptoms of CMD in bruxers classified by the degree of severity. Cranio. 1999;17(4):268-79. PMid:10650399

17. Buysse DJ, Reynolds CF 3rd, Monk TH, Berman SR, Kupfer DJ.ThePittsburg Sleep Quality Index: a newinstrumentfor psychiatric practice and research. Psychiatry Res. 1989; 28(2):193-213. doi:10.1016/0165-1781(89)90047-4.

18. Cardoso HC, Bueno FCC, Mata JC, Alves APR, Jochims I, Filho IHRV, et al. Avaliação da qualidade do sono em estudantes de Medicina. Rev Bras Educ Med. 2009;33(3):34955. doi:10.1590/S0100-55022009000300005.

19. Branco RS, Branco CS, Tesch RS, Rapoport A. Freqüência de relatos de parafunções nos subgrupos diagnósticos de DTM de acordo com os critérios diagnósticos para pesquisa em disfunções temporomandibulares (RDC/ TMD). R Dental Press Ortodon Ortop Facial. 2008; 13(2):61-9. doi:10.1590/S1415-54192008000200008.

20. Seraidarian PI, Assunção ZLV, Jacob MF. Bruxismo: uma atualização dos conceitos, etiologia, prevalência e gerenciamento. JBA. 2001;1(4):290-5.
21. Molina OF, Sobreira MA, Tavares PG, Dib JE, Aquilino RN. Anxiety in craniomandibular disorders and bruxing behavior patients: the role of pain in single and multiple sites and severity of bruxing behavior. Part I: inclusion of the extreme behavior group and report of preliminary psychological data on anxiety. Rev Neurocienc. 2006;14(1):23-30.

22. Verri FR, Garcia AR, Zuim PRJ, Almeida EO, Falcón-Antenucci RM, Shibayama R. Avaliação da qualidade do sono em grupos com diferentes níveis de desordem temporomandibular. Pesq Bras Odontoped Clin Integr. 2008;8(2):165-9. doi:10.4034/1519.0501.20 08.0082.0006.

23. Lockley SW, Skene DJ, Arendt J, Tabandeh H, Bird AC, Defrance R. Relationship between melatonin rhythms and visual loss in the blind. J Clin Endocrinol Metab. 1997;82(11):3763-70. doi:10.1210/jc.82.11.3763.

24. Leger D, Guilleminault C, Defrance R, Domont A, Paillard M. Prevalence of sleep/wake disorders in persons with blindness. Clinical Science. 1999;97:193-9. doi:10.1042/CS19990004.

25. Pierce CJ, Chrisman K, Bennett ME, Close JM. Stress, anticipatory stress and psychologic measures related to sleep bruxism. J Orofac Pain. 1995;9(1):51-6. PMid:7581205.

26. Lavigne GJ, Lobbezoo F, Rompre PH, Nielsen TA, Montplaisir JY. Cigarette smoking as a risk factor or an exacerbating factor for restless legs syndrome and sleep bruxism. Sleep. 1997;20(4):290-3. PMid:9231955.

27. Aloé F, Gonçalves LR, Azevedo A, Barbosa RC. Bruxismo durante o sono. Rev Neurociências. 2003;11(1):4-17.

28. Jesus LA. Efeito da placa de Michigan sobre o sinal eletromiográfico e dor em pacientes bruxistas com DTM: ensaio clínico controlado e randomizado [dissertação de mestrado]. São Paulo: Universidade Nove de Julho; 2008.

29. Costa LFM, Guimarães JP, Chaobah A. Prevalência de distúrbios da articulação têmporo-mandibular em crianças e adolescentes brasileiros e sua relação com má-oclusão e hábitos parafuncionais: um estudo epidemiológico transversal - parte II: distúrbios articulares e hábitos para funcionais. J Bras Ortodon Ortop Facial. 2004;9(50):162-9. 
da Silva BBR, Lacerda KRRS, Ferreira APL, Figueiroa MS.

30. Watanabe T, Ichikawa K, Clark GT. Bruxism levels and daily behaviors: 3 weeks of measurement and correlation. J Orofac Pain. 2003;17(1):65-73. PMid:12756933.

31. Waller EA, Bendel RE, Kaplan J. Sleep disorders and the eye. Mayo Clin Proc. 2008;83(11):1251-61. doi:10.4065/83.11.1251.

Recebido: 27/04/2012

Received: 04/27/2012

Aprovado: 28/12/2012

Approved: 12/28/2012 